\title{
Measurement-Based Variational Quantum Eigensolver
}

\author{
R. R. Ferguson $\odot,{ }^{1, *}$ L. Dellantonio $\odot,{ }^{1, *}$ A. Al Balushi, ${ }^{1}$ K. Jansen, ${ }^{2}$ W. Dür ${ }^{3},{ }^{3}$ and C. A. Muschik $\oplus^{1,4}$ \\ ${ }^{1}$ Institute for Quantum Computing and Department of Physics and Astronomy, \\ University of Waterloo, Waterloo N2L 3G1, Canada \\ ${ }^{2}$ NIC, DESY Zeuthen, Platanenallee 6, 15738 Zeuthen, Germany \\ ${ }^{3}$ Institut für Theoretische Physik, Universität Innsbruck, Technikerstraße 21a, 6020 Innsbruck, Austria \\ ${ }^{4}$ Perimeter Institute for Theoretical Physics, Waterloo, Ontario N2L 2Y5, Canada
}

(Received 5 November 2020; revised 8 March 2021; accepted 9 March 2021; published 1 June 2021)

\begin{abstract}
Variational quantum eigensolvers (VQEs) combine classical optimization with efficient cost function evaluations on quantum computers. We propose a new approach to VQEs using the principles of measurement-based quantum computation. This strategy uses entangled resource states and local measurements. We present two measurement-based VQE schemes. The first introduces a new approach for constructing variational families. The second provides a translation of circuit- to measurement-based schemes. Both schemes offer problem-specific advantages in terms of the required resources and coherence times.
\end{abstract}

DOI: 10.1103/PhysRevLett.126.220501

Variational methods are crucial to investigate the physics of strongly correlated quantum systems. Numerical tools like the density matrix renormalization group [1-4] have been applied to several problems, including real-time dynamics [5], condensed matter physics [6], lattice gauge theories [7-10], and quantum chemistry [11,12]. Nevertheless, the classes of states that can be studied with classical computers are limited [13]. Variational quantum eigensolvers (VQEs) overcome this problem using a closed feedback loop between a classical computer and a quantum processor [14-16]. The classical algorithm optimizes a cost function-typically the expectation value of some operator-which is efficiently supplied by the quantum hardware. The VQE provides an approximation to the (lowlying) eigenvalues of this operator and the corresponding eigenstates. VQEs are advantageous for a variety of applications [16-22] and have been experimentally demonstrated in fields including chemistry [23,24], particle physics [25-28], and classical optimization [29-31].

Existing VQE protocols are based on the circuit model, where gates are applied on an initial state [32]. These gates involve variational parameters whose optimization allows the resulting output state to approximate the desired target state. We propose a new approach to VQE protocols, based on the measurement-based model of quantum computation (MBQC) [33-38]. In MBQC, an entangled state is prepared and the computation is realized by performing single-qubit measurements. While the circuit- and measurement-based models both allow for universal quantum computation and have equivalent scaling of resources [36], they are intrinsically different. The former is limited by the number of available qubits and gates that can be performed, and MBQC is limited by the size of the entangled state one can generate. For certain applications, the required coherence times $[39,40]$ and error thresholds [36,39-41] are much less demanding for MBQC.

Here, we develop a new variational technique based on MBQC, that we call measurement-based VQE (MB-VQE). Our protocols determine the ground state of a target Hamiltonian, which is a prototypical task for VQEs with wide-ranging applications [14,20-23,26,42]. The underlying idea is to use a tailored entangled state called a "custom state," that allows for exploring an appropriate corner of the system's Hilbert space [Fig. 1(a)]. This custom state includes auxiliary qubits, which, once measured, modify the state of the output qubits. The choices of the measurement bases and the corresponding variational changes to the state are controlled by a classical optimization algorithm. This approach differs conceptually and practically from standard VQE schemes since MB-VQE shifts the challenge from performing multiqubit gates to creating an entangled initial state.

After presenting the framework for the $\mathrm{MB}-\mathrm{VQE}$, we design two specific schemes that are suited to different problem classes. First, we introduce a novel method to construct variational state families, illustrated using the toric code model with local perturbations [43]. As Fig. 1(a) shows, we start from an ansatz state $\left|\psi_{a}\right\rangle$ in an appropriate corner of the Hilbert space. To explore this neighborhood using a classical optimization algorithm, we introduce a custom state and apply measurement-based modifications of $\left|\psi_{a}\right\rangle$ that have no direct analog in the circuit model. The resulting variational family is not efficiently accessible with known classical methods and is more costly to access with circuit-based VQEs. 

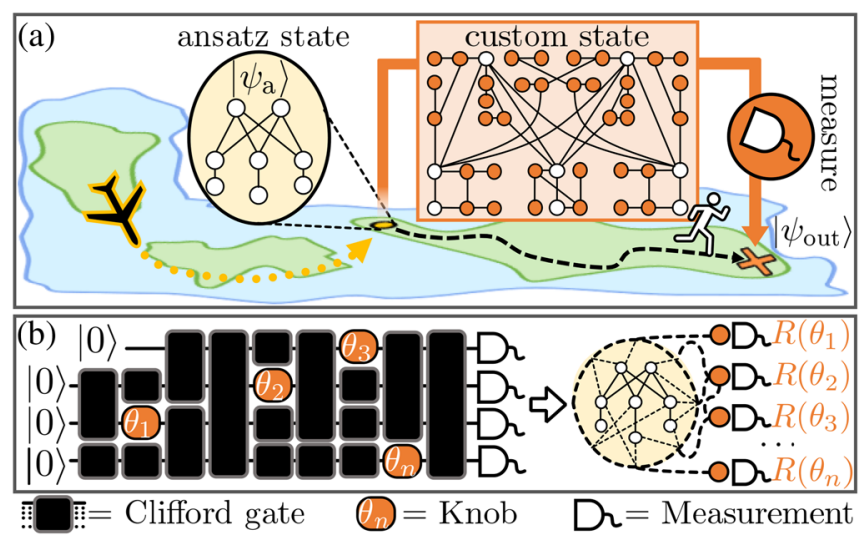

FIG. 1. MB-VQE schemes. (a) Variation of a problem-specific ansatz state by "edge decoration." An ansatz graph state starts the MB-VQE in a suitable corner of Hilbert space (choice of green island). Next, a classical algorithm explores the neighborhood (runner on black arrow). The variational optimization exploits a custom state that is obtained by decorating the edges of the ansatz state with auxiliary qubits (orange circles). Their measurement in rotated bases $R(\theta)$ with variational parameters $\theta$ transforms $\left|\psi_{a}\right\rangle$ into the output state $\left|\psi_{\text {out }}\right\rangle$. (b) Direct translation of a VQE circuit into a MB-VQE. Left: circuit consisting of Clifford gates (black) and single-qubit parametric gates ("knobs"). Right: corresponding MB-VQE, where the Clifford part of the circuit has been performed beforehand. The custom state consists of output (white circles) and auxiliary (orange circles) qubits only; the latter are measured in rotated bases and are related to the knobs in the circuit.

Second, we introduce a direct translation of circuit VQEs to MB-VQEs [Fig. 1(b)]. Here, the variational state family is the same for the circuit- and measurement-based approaches, but the implementation differs as the MBVQE requires different resources and is manipulated by single-qubit measurements only. We exemplify this direct translation for the Schwinger model [44] and highlight the different hardware requirements and the scaling of resources. As explained below, a translation to MB-VQE is advantageous for circuits containing a large fraction of socalled Clifford gates (e.g., $C X$ gates), as these are absorbed into the custom state.

While MB-VQE is platform agnostic, it opens the door for complex quantum computations in systems where long gate sequences or the realization of entangling gates are challenging. In particular, MB-VQE offers new routes for experiments with photonic quantum systems, thus enlarging the toolbox of variational computations.

General framework.-The main resource of MBQC are so-called graph states $[45,46]$. Graphs as in Fig. 1 are stabilizer states (eigenstates with +1 eigenvalues) of the operators $\hat{S}_{n}=\hat{X}_{n} \prod_{k} \hat{Z}_{k}$, where $k$ refers to the vertices connected to site $n$. To obtain the desired final state encoded in the output qubits (white circles), singlequbit measurements are performed on auxiliary qubits (orange circles), either in the eigenbasis of the Pauli operators $\hat{X}, \hat{Y}, \hat{Z}, \quad$ or in the rotated basis $R(\theta) \equiv\left\{\left(|0\rangle \pm e^{i \theta}|1\rangle\right) / \sqrt{2}\right\}$. Depending on the measurement outcomes, the system is probabilistically projected into different states. To make the computation deterministic, so-called by-product operators and adaptive measurements are required [36]. The former applies $\hat{X}$ and $\hat{Z}$ operators to the output qubits depending on the measurement results, while the latter involves adapting the measurement bases $R(\theta)$ based on earlier measurement outcomes. Consequently, adaptive measurements must be performed in a specific order.

An advantage of MBQC is the possibility to simultaneously perform all nonadaptive measurements at the beginning of the calculation [see Fig. 1(b) and Supplemental Material [47]]. This corresponds to the Clifford part of a circuit and includes single- and manyqubit gates. This is independent of the position of the gates in the circuit, and reduces the required overhead and coherence time. Remarkably, this can be either done directly on the graph state in the quantum hardware or on a classical computer before the experiment. In the latter case, the Gottesman-Knill theorem [48] allows for efficiently determining the custom state which is local-Clifford equivalent to the quantum state obtained after all nonadaptive measurements are performed [50]. This state can be directly prepared and used for the MBQC, which may have dramatically fewer auxiliary qubits compared to the initial graph state.

We now explain how MBQC is used to design a MB-VQE. While the classical part of the feedback loop is untouched (the best optimization algorithm [51-56] is problem dependent $[26,57])$, the MB-VQE is based on the creation and partial measurement of a tailored graph state rather than the application of a sequence of gates. Specifically, the quantum part of a MB-VQE comprises an ansatz state $\left|\psi_{a}\right\rangle$, a custom state, and a measurement prescription. As schematically represented in Fig. 1(a), $\left|\psi_{a}\right\rangle$ is a graph state from which we start exploring the variational class of families attainable by the MB-VQE. The custom state is then created by expanding $\left|\psi_{a}\right\rangle$ into a bigger graph state. This is done by decoration, i.e., by adding new vertices and connecting them to preexisting sites in the ansatz state. According to a measurement prescription, which is the same at each iteration of the algorithm, the auxiliary qubits of the custom state are then measured, with the remaining ones constituting the output $\left|\psi_{\text {out }}\right\rangle$ of the quantum processor [see Fig. 1(a)]. The cost function to be fed into the classical side of the MB-VQE is then calculated from $\left|\psi_{\text {out }}\right\rangle$ (e.g., its energy), with the angles $\theta$ of the rotated bases $R(\theta)$ being the variational parameters over which the optimization occurs.

Just like the circuit in a VQE, the custom state determines the success of our MB-VQE. Generally, the more auxiliary qubits that are measured in rotated bases $R(\theta)$, the bigger the available class of variational states that can be 
explored. However, an excessive number of parameters $\theta$ makes the algorithm's convergence slower. Therefore, it is convenient to tailor the custom state to the considered problem. Qubit decoration, with the subsequent measurement of the auxiliary qubits, allows for remarkable control over the desired ansatz state's transformation(s). Not only can one apply gates-just like in a circuit-based VQE-by following MBQC prescriptions (see the Schwinger model example), one can also identify completely new patterns of auxiliary qubits that modify the output state in a way that would be expensive or even impossible with the circuit formalism (see the toric code example). For instance, a single auxiliary qubit measured in $R(\theta)$ and connected to an arbitrary number of output qubits $\{1,2,3, \ldots\}$, acts $e^{i(\theta / 2) \hat{Z}_{1} \otimes \hat{Z}_{2} \otimes \hat{Z}_{3} \otimes \cdots}$ onto them [35]. In a circuit, the same operation requires a linear number of 2-qubit gates [58].

State variation by edge modification (perturbed toric code).-Here, we demonstrate how a MB-VQE manipulates states in a different way than a circuit-based VQE. MB-VQEs are advantageous whenever a perturbation $\hat{H}_{p}$ is added to a Hamiltonian $\hat{H}_{0}$, whose ground state, used as ansatz state $\left|\psi_{a}\right\rangle$ below, is a stabilizer state or a graph state.

To create the custom state from $\left|\psi_{a}\right\rangle$, we employ the pattern in Fig. 2(a), which decorates each connected pair of output qubits $m$ and $n$ with four auxiliary qubits $(m, n)_{i}$ $(i=1, \ldots, 4)$, to be measured in rotated bases $R(\theta)$. Depending on $R(\theta)$, the entanglement between the qubits $m$ and $n$ is modified, and their state is subjected to an
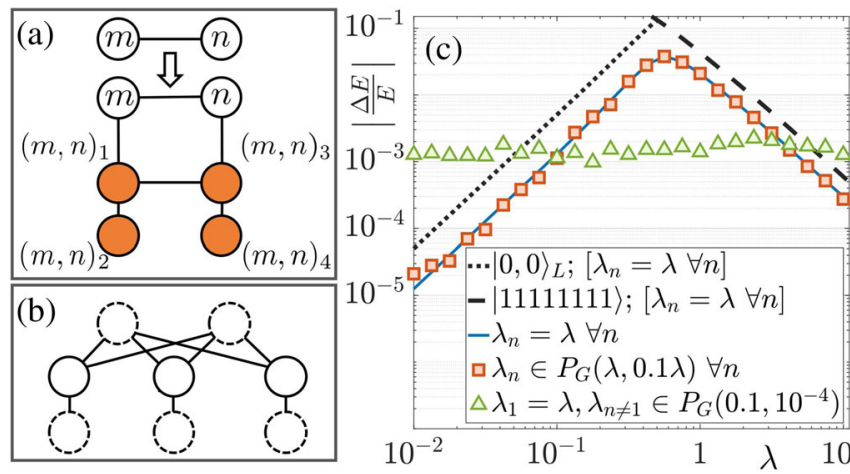

FIG. 2. Perturbed toric code. (a) Edge modification resource for the MB-VQE [see Fig. 1(a)]. Four auxiliary qubits (orange circles), labeled $(m, n)_{i}(i=1, \ldots, 4)$, are added to two connected output qubits $m$ and $n$ (white circles). (b) Graph state representation of the ansatz state $|0,0\rangle_{L}$. Additional Hadamard gates are applied to qubits with dashed lines. (c) Relative difference between the MB-VQE result and the true ground state energy vs the perturbation strength. We let $\lambda_{n}$ in Eq. (1) be equal on all qubits (solid blue line) or sampled from a normal distribution $P_{G}$ of average $\lambda$ and variance $0.1 \lambda$ (red squares). Green triangles describe a perturbation acting strongly on $\lambda_{1}$ and weakly on the other qubits. Dotted and dashed lines are computed with respect to $|0,0\rangle_{L}$ (ansatz state) and $|1\rangle^{\otimes 2 N_{x} N_{y}}$ (ground state of $\hat{H}_{p}$ ) for $\lambda_{n}=\lambda \forall n$. additional rotation. For example, if all auxiliary qubits in the custom state are measured with $\theta=0$, we obtain the original ansatz state. However, if all auxiliary qubits are measured with $\theta=\pi / 2$, then all entanglement of $\left|\psi_{a}\right\rangle$ is eliminated (for more details, see Supplemental Material [47]). This decoration technique is tailored to the perturbed toric code example below, in which the ground states of $\hat{H}_{0}$ and $\hat{H}_{p}$ are maximally entangled and pure, respectively. However, it can be easily generalized to expand the class of available variational states (see Supplemental Material [47]), thus suiting different scenarios.

We apply this MB-VQE approach to the toric code model, a quantum error-correcting code defined on a twodimensional rectangular lattice with periodic boundary conditions [59]. On the lattice, the number of rows (columns) of independent vertices is $N_{x}\left(N_{y}\right)$ and edges represent qubits. The toric code state is a stabilizer state of so-called star $\hat{A}_{s}$ and plaquette $\hat{B}_{p}$ operators. For any vertex $s$ in the lattice, $\hat{A}_{s}$ acts $\hat{Z}$ on the four incident edges, while $\hat{B}_{p}$ acts $\hat{X}$ on the four edges in the $p$ th plaquette. The toric code Hamiltonian is then $\hat{H}_{0}=-\sum_{s} \hat{A}_{s}-\sum_{p} \hat{B}_{p}$. Since $\prod_{s} \hat{A}_{s}=\prod_{p} \hat{B}_{p}=1$, the toric code has $2 N_{x} N_{y}-2$ independent stabilizers, and $\hat{H}_{0}$ has four degenerate ground states $|r, t\rangle_{L}(r, t=0,1)$, called logical states below. These are simultaneous eigenstates of $\hat{H}_{0}$ and the two logical- $Z$ operators [59], as explained in the Supplemental Material [47].

The perturbation added to the toric code Hamiltonian is

$$
\hat{H}_{p}=\sum_{n=1}^{2 N_{x} N_{y}} \lambda_{n} \hat{Z}_{n},
$$

which corresponds to an inhomogeneous magnetic field. As ansatz state for the MB-VQE, we choose the highly entangled graph state $\left|\psi_{a}\right\rangle=|0,0\rangle_{L}$ that approximates the ground state of $\hat{H}_{0}+\hat{H}_{p}$ for small positive values of $\lambda_{n}$. The graph state representation of $|0,0\rangle_{L}$ can be calculated efficiently classically [50] and is shown in Fig. 2(b) for $N_{x}=N_{y}=2$. In the Supplemental Material [47], we explain how to adapt our MB-VQE protocol to use an arbitrary superposition of $|r, t\rangle_{L}(r, t=0,1)$ as ansatz state, which is more suited for different kinds of perturbations $\hat{H}_{p}$.

Numerical results for the $\mathrm{MB}-\mathrm{VQE}$ are shown in Fig. 2(c). The relative energy difference between the MB-VQE result and the true ground state (calculated via exact diagonalization) is plotted against the perturbation strength. This is done with all $\lambda_{n}$ in Eq. (1) equal to $\lambda$ (solid blue line), with each $\lambda_{n}$ drawn from a Gaussian distribution $P_{G}\left(\mu, \sigma^{2}\right)$ with mean $\mu=\lambda$ and variance $\sigma^{2}=0.1 \lambda$ (orange squares), and with $\lambda_{1}=\lambda, \lambda_{n}$ randomly sampled from $P_{G}\left(\mu=0.1, \sigma^{2}=10^{-4}\right)$ for $n \neq 1$ (green triangles). A plot of the infidelity resembles Fig. 2(c), with maximum 
infidelities for the blue curve, red squares, and green triangles being $6.2 \times 10^{-2}, 6.5 \times 10^{-2}$, and $9.4 \times 10^{-3}$, respectively. Figure 2(c) shows that the MB-VQE produces the ground state energy with high confidence when the perturbation strength is very small or very large. Notably, the MB-VQE outperforms the ansatz state (dotted black line) and the ground state of $\hat{H}_{p}$ in Eq. (1) (dashed black line) in all cases. If the perturbation only acts on one qubit, the chosen custom state allows the MB-VQE to find the exact ground state energy within machine precision. This is also the case if the perturbation acts on two disconnected qubits, provided we connect them and add auxiliary qubits as in Fig. 2(a). This suggests that the outcome of the MB-VQE can be significantly improved by adding few extra auxiliary qubits.

Translating VQEs into MB-VQEs (Schwinger model).Instead of the approach described above, one can create a MB-VQE by translating the circuit of a VQE into its corresponding custom state and a sequence of measurements. Since a universal set of gates can be realized in a MBQC [36], any VQE can be translated into a MB-VQE. As we discuss below, this strategy is advantageous if the number of parametric adaptive measurements [i.e., knobs in Fig. 1(b)] in the resulting MB-VQE scheme is small.

As an example, we determine the ground state energy of the Schwinger model [44], a test bed used for benchmarking quantum simulations in high-energy physics $[8,25,60]$. The Schwinger model describes quantum electrodynamics on a one-dimensional lattice and can be cast in the form of a spin model with long-range interactions [61-63],

$$
\begin{aligned}
\hat{H}= & \frac{J}{2} \sum_{n=1}^{S-2} \sum_{k=n+1}^{S-1}(S-k) \hat{Z}_{n} \hat{Z}_{k}-\frac{J}{2} \sum_{n=1}^{S-1} n \bmod 2 \sum_{k=1}^{n} \hat{Z}_{k} \\
& +w \sum_{n=1}^{S-1}\left(\hat{\sigma}_{n}^{+} \hat{\sigma}_{n+1}^{-}+\text {H.c. }\right)+\frac{\mu}{2} \sum_{n=1}^{S}(-1)^{n} \hat{Z}_{n},
\end{aligned}
$$

where $S$ is the number of fermions, $\mu$ is their mass, $w=1 / 2 a$, and $J=\left(g^{2} a / 2\right)$. Here, $a$ and $g$ are the lattice spacing and the coupling strength, respectively, and $\hat{\sigma}_{n}^{ \pm}=\left(\hat{X}_{n} \pm i \hat{Y}_{n}\right) / 2$.

For the VQE protocol, we assume the typical situation where parametric single-qubit gates and fixed entangling gates $(C X \mathrm{~s})$ are used $[23,64]$. We consider a generic VQE circuit, in which a sequence of "layers" is applied [14], each containing local rotations and entangling gates. As shown in Fig. 3(a) for $S=4$, we choose the layer

$$
\prod_{n=1}^{S / 2-1} C X_{2 n, 2 n+1} \prod_{n=1}^{S / 2} C X_{2 n-1,2 n} \prod_{n=1}^{S} \hat{U}_{x, n}\left(\theta_{x, n}\right) \hat{U}_{z, n}\left(\theta_{z, n}\right)
$$

where $\hat{U}_{\nu, n}\left(\theta_{\nu, n}\right)=\exp \left(i \theta_{\nu, n} \hat{V}_{n} / 2\right) \quad[(\nu, \hat{V})=(x, \hat{X})$ or $(\nu, \hat{V})=(z, \hat{Z})]$. The circuit for the $\mathrm{VQE}$ is created by concatenating $K$ layers, where $K$ is big enough to
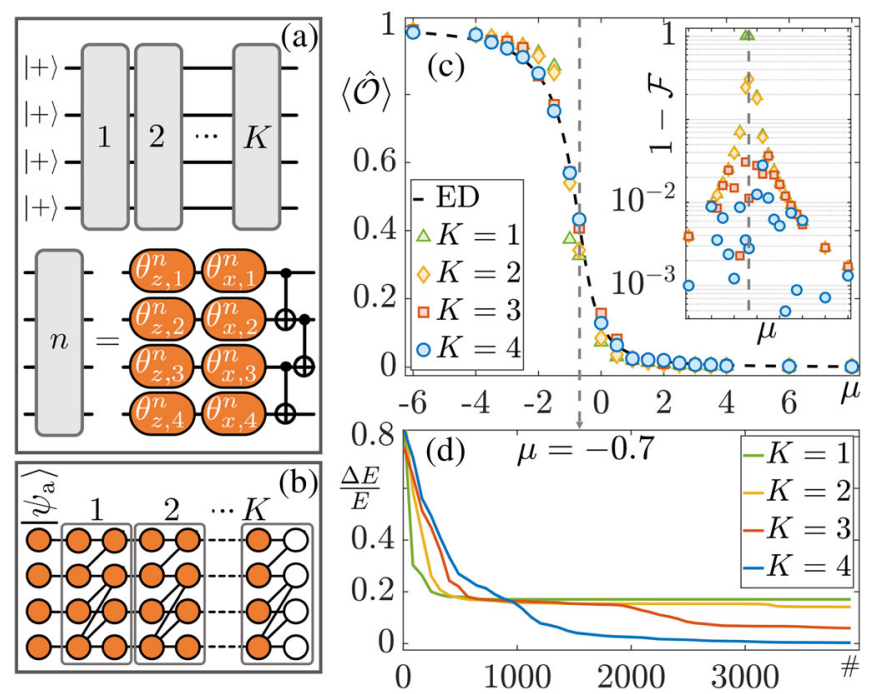

FIG. 3. Schwinger model. (a) Ansatz state and VQE circuit for $S=4$ qubits and $K$ layers. Each layer consists of $C X$ gates and local rotations (orange) parametrized by angles $\theta_{\nu, i}^{n}$ (with rotation axis $\nu=x, z ; i=1, \ldots, 4)$. (b) MB-VQE custom state for $K$ layers. White circles are output qubits. Auxiliary qubits (orange) are measured in a rotated bases $R(\theta)$. (c) The order parameter $\langle\hat{\mathcal{O}}\rangle$ vs the fermion mass $\mu$. The dashed line and dots represent exact diagonalization (ED) and (MB-)VQE results, respectively, with the number of layers $K$ indicated in the legend. The inset shows the infidelity $1-\mathcal{F}$. (d) Relative energy difference $\Delta E / E$ between (MB-)VQE results and $\mathrm{ED}$ for $\mu=-0.7$ vs the number of iterations in the optimization procedure. The variational parameters are initialized at zero, and $J=\omega=1$ in Eq. (2).

sufficiently explore the relevant subsector of the considered Hilbert space. As described in the Supplemental Material [47], the MB-VQE custom state corresponding to a $K$-layer circuit is obtained by joining the measurement patterns of the gates in Eq. (3) and performing all nonadaptive measurements classically, which effectively removes the Clifford parts of the circuit. The custom state is shown in Fig. 3(b). As ansatz state we use $\left|\psi_{a}\right\rangle=\stackrel{S}{\bigotimes}|+\rangle$.

The (MB-)VQE simulation results are shown in Fig. 3(c) for $S=4$ and different values of $K$. We plot the order parameter $\langle\hat{\mathcal{O}}\rangle=[1 / 2 S(S-1)] \sum_{i, j<i}\left\langle\left[1+(-1)^{i} \hat{Z}_{i}\right]\right.$ $\left.\left[1+(-1)^{j} \hat{Z}_{j}\right]\right\rangle$ against the fermion mass $\mu$ and correctly observe a second-order phase transition around $\mu=-0.7$ $[25,65,66]$. Increasing $K$ improves the ground state approximation, as demonstrated by the inset in Fig. 3(c) and by Fig. 3(d). The points near the phase transition require $K \gtrsim 3$ layers ( $\gtrsim 28$ qubits), whereas $K=1$ layer (12 qubits) suffices for the easiest points. Note that allowing different gates as resources in Eq. (3) generally leads to different convergence rates, as demonstrated by the results in Ref. [25].

Perfect platforms provided, both the VQE and the MB-VQE give the same result. However, the quantum hardware requirements are different for the two methods. 
The circuit-based VQE requires $S$ qubits, $2 K S$ single-qubit operations, and $K(S-1)$ entangling gates. For the corresponding MB-VQE, a custom state of $S(2 K+1)$ qubits and $2 K S$ single-qubit operations (measurements) are required. Generally, translating a VQE into its corresponding MB-VQE is advantageous whenever the VQE circuit involves a large Clifford part compared to the number of adaptive measurements (i.e., knobs). In this case, MB-VQE avoids the requirement of performing long gate sequences, which is currently challenging due to error accumulation. This is especially interesting for platforms where entangling gates are hard to realize (e.g., photonic setups) or in systems with limited coherence times.

Conclusions. - In this Letter, we merged the principles of measurement-based quantum computation and quantumclassical optimization to create a MB-VQE. We presented two new types of variational schemes that are applicable but not restricted to the examples given. The first applies when the ansatz state is a stabilizer state. In this case, it is classically efficient to determine the corresponding graph state [50], which is decorated with additional control qubits and prepared directly. We applied this MB-VQE to the perturbed toric code. Additionally, we showed how to adapt any circuit-based VQE to become a MB-VQE, with the Schwinger model as example.

Experimental proof-of-concept demonstrations can be explored by considering the smallest instance of the planar code [59] with a perturbation on a single qubit as first step. In this scenario, the MB-VQE requires as few as eight entangled qubits instead of the 44 used above. Especially promising candidate systems include superconducting qubits and photonic platforms. The latter recently demonstrated the capability to entangle several thousands of qubits $[67,68]$ and to create tailored graph states [69-72]. When designing custom states for future experiments, it will be important to understand the effects of decoherence and it will be interesting to investigate whether MB-VQEs retain the high robustness of MBQC against errors [39-41].

Our scheme based on edge decoration provides a new way of thinking about state variations in VQEs. In particular, the effects resulting from measuring only one or few entangled auxiliary qubits can be challenging to describe with a simple circuit. The resulting state modifications do not necessarily correspond to unitary operations and can affect a large number of remaining qubits [35]. Accordingly, MB-VQEs can lead to schemes in which few auxiliary qubits suffice to reach the desired state, while many gates would be required in a circuit-based protocol. Just like circuit optimization in standard VQEs [73], tailored decorations can lead to more resource-efficient MB-VQEs, with the custom state optimized to the specific problem. The framework presented here provides a starting point for designing VQEs whose properties are different and complementary to the standard approach that is based on varying a state by applying gates.
We thank Joachim von Zanthier, Jinglei Zhang, and Ralf Röhlsberger for useful discussions. This work was supported by the Transformative Quantum Technologies Program (CFREF), NSERC and the New Frontiers in Research Fund, the Institute of Quantum Computing (IQC), and the Austrian Science Fund (FWF) through Project No. P30937N27. We also thank the Army Research Laboratory's Center for Distributed Quantum Information, Cooperative Agreement No. W911NF15-2-0060. C. M. received support from the Alfread P. Sloan Foundation in the form of a Sloan Research Fellowship.

${ }^{*}$ R. R. F. and L. D. contributed equally to this work.

[1] D. Perez-Garcia, F. Verstraete, M. Wolf, and J. Cirac, Quantum Inf. Comput. 7, 401 (2007).

[2] J. Haegeman, T. J. Osborne, and F. Verstraete, Phys. Rev. B 88, 075133 (2013).

[3] R. Orús, Eur. Phys. J. B 87, 280 (2014).

[4] G. Evenbly and G. Vidal, Phys. Rev. Lett. 115, 180405 (2015).

[5] F. Güttge, F. B. Anders, U. Schollwöck, E. Eidelstein, and A. Schiller, Phys. Rev. B 87, 115115 (2013).

[6] Y.-C. He, M. P. Zaletel, M. Oshikawa, and F. Pollmann, Phys. Rev. X 7, 031020 (2017).

[7] M. C. Bañuls, R. Blatt, J. Catani, A. Celi, J. I. Cirac, M. Dalmonte, L. Fallani, K. Jansen, M. Lewenstein, S. Montangero, C. A. Muschik, B. Reznik, E. Rico, L. Tagliacozzo, K. Van Acoleyen, F. Verstraete, U.-J. Wiese, M. Wingate, J. Zakrzewski, and P. Zoller, Eur. Phys. J. D 74, 165 (2020).

[8] M. C. Bañuls and K. Cichy, Rep. Prog. Phys. 83, 024401 (2020).

[9] M. Dalmonte and S. Montangero, Contemp. Phys. 57, 388 (2016).

[10] J. Bender, P. Emonts, E. Zohar, and J. I. Cirac, Phys. Rev. Research 2, 043145 (2020).

[11] G. K.-L. Chan, J. J. Dorando, D. Ghosh, J. Hachmann, E. Neuscamman, H. Wang, and T. Yanai, in Frontiers in Quantum Systems in Chemistry and Physics, Progress in Theoretical Chemistry and Physics Vol. 18, edited by S. Wilson, P. J. Grout, J. Maru-ani, G. Delgado-Barrio, and P. Piecuch (Springer, New York, 2008), pp. 49-65.

[12] C. Krumnow, L. Veis, O. Legeza, and J. Eisert, Phys. Rev. Lett. 117, 210402 (2016).

[13] G. Ortiz, J. E. Gubernatis, E. Knill, and R. Laflamme, Phys. Rev. A 64, 022319 (2001).

[14] J. R. McClean, J. Romero, R. Babbush, and A. Aspuru-Guzik, New J. Phys. 18, 023023 (2016).

[15] E. Farhi, J. Goldstone, and S. Gutmann, Report No. MIT-CTP/4610, 2014.

[16] J. Preskill, Quantum 2, 79 (2018).

[17] O. Shehab, I. H. Kim, N.H. Nguyen, K. Landsman, C. H. Alderete, D. Zhu, C. Monroe, and N. M. Linke, arXiv:1906.00476.

[18] M. C. Bañuls, R. Blatt, J. Catani, A. Celi, J. I. Cirac, M. Dalmonte, L. Fallani, K. Jansen, M. Lewenstein, S. Montangero et al., Eur. Phys. J. D 74, 165 (2020). 
[19] R. Kaubruegger, P. Silvi, C. Kokail, R. van Bijnen, A. M. Rey, J. Ye, A. M. Kaufman, and P. Zoller, Phys. Rev. Lett. 123, 260505 (2019).

[20] E. F. Dumitrescu, A. J. McCaskey, G. Hagen, G. R. Jansen, T. D. Morris, T. Papenbrock, R. C. Pooser, D. J. Dean, and P. Lougovski, Phys. Rev. Lett. 120, 210501 (2018).

[21] J. F. Haase, L. Dellantonio, A. Celi, D. Paulson, A. Kan, K. Jansen, and C. A. Muschik, Quantum 5, 393 (2021).

[22] B. Bauer, D. Wecker, A. J. Millis, M. B. Hastings, and M. Troyer, Phys. Rev. X 6, 031045 (2016).

[23] S. McArdle, S. Endo, A. Aspuru-Guzik, S. C. Benjamin, and X. Yuan, Rev. Mod. Phys. 92, 015003 (2020).

[24] P. J. J. O’Malley, R. Babbush, I. D. Kivlichan, J. Romero, J. R. McClean, R. Barends, J. Kelly, P. Roushan, A. Tranter, N. Ding et al., Phys. Rev. X 6, 031007 (2016).

[25] C. Kokail, C. Maier, R. van Bijnen, T. Brydges, M. K. Joshi, P. Jurcevic, C. A. Muschik, P. Silvi, R. Blatt, C. F. Roos et al., Nature (London) 569, 355 (2019).

[26] D. Paulson, L. Dellantonio, J. F. Haase, A. Celi, A. Kan, A. Jena, C. Kokail, R. van Bijnen, K. Jansen, P. Zoller et al., arXiv:2008.09252.

[27] O. Shehab, K. Landsman, Y. Nam, D. Zhu, N. M. Linke, M. Keesan, R. C. Pooser, and C. Monroe, Phys. Rev. A $\mathbf{1 0 0 ,}$ 062319 (2019).

[28] H.-H. Lu, N. Klco, J. M. Lukens, T. D. Morris, A. Bansal, A. Ekström, G. Hagen, T. Papenbrock, A. M. Weiner, M. J. Savage, and P. Lougovski, Phys. Rev. A 100, 012320 (2019).

[29] A. Borle, V. E. Elfving, and S. J. Lomonaco, arXiv:2006.15438.

[30] C. Bravo-Prieto, R. LaRose, M. Cerezo, Y. Subasi, L. Cincio, and P. Coles, Bull. Am. Phys. Soc. 65 (2020).

[31] O. Kyriienko, A. E. Paine, and V. E. Elfving, arXiv:2011.10395.

[32] A. Peruzzo, J. McClean, P. Shadbolt, M.-H. Yung, X.-Q. Zhou, P. J. Love, A. Aspuru-Guzik, and J. L. O'Brien, Nat. Commun. 5, 4213 (2014).

[33] R. Raussendorf and H. J. Briegel, Phys. Rev. Lett. 86, 5188 (2001).

[34] H. J. Briegel, D. E. Browne, W. Dür, R. Raussendorf, and M. Van den Nest, Nat. Phys. 5, 19 (2009).

[35] D. E. Browne and H. J. Briegel, arXiv:quant-ph/0603226.

[36] R. Raussendorf, D. E. Browne, and H. J. Briegel, Phys. Rev. A 68, 022312 (2003).

[37] P. Walther, K. J. Resch, T. Rudolph, E. Schenck, H. Weinfurter, V. Vedral, M. Aspelmeyer, and A. Zeilinger, Nature (London) 434, 169 (2005).

[38] M. V. Larsen, X. Guo, C. R. Breum, J. S. NeergaardNielsen, and U. L. Andersen, arXiv:2010.14422.

[39] M. Zwerger, H. Briegel, and W. Dür, Appl. Phys. B 122, 50 (2016).

[40] M. Zwerger, H. Briegel, and W. Dür, Sci. Rep. 4, 5364 (2014).

[41] M. Zwerger, H. J. Briegel, and W. Dür, Phys. Rev. Lett. 110, 260503 (2013).

[42] N. Moll, P. Barkoutsos, L. S. Bishop, J. M. Chow, A. Cross, D. J. Egger, S. Filipp, A. Fuhrer, J. M. Gambetta, M. Ganzhorn et al., Quantum Sci. Technol. 3, 030503 (2018).

[43] R. Hübener, C. Kruszynska, L. Hartmann, W. Dür, M. B. Plenio, and J. Eisert, Phys. Rev. B 84, 125103 (2011).

[44] J. Schwinger, Phys. Rev. 82, 914 (1951).
[45] M. Hein, J. Eisert, and H. J. Briegel, Phys. Rev. A 69 , 062311 (2004).

[46] M. Hein, W. Dür, J. Eisert, R. Raussendorf, M. Nest, and H.-J. Briegel, Proceedings of the International School of Physics "Enrico Fermi" on Quantum Computers, Algorithms and Chaos, edited by G. Casati, D. L. Shepelyansky, P. Zoller, and G. Benenti (IOS Press, 2006), Vol. 162 [arXiv:quant-ph/0602096].

[47] See Supplemental Material at http://link.aps.org/supplemental/ 10.1103/PhysRevLett.126.220501 for discussions on decorated edges, logical- $Z$ and logical- $X$ operators for the toric code, creating entangled superpositions of the toric code ground states, and combining measurement patterns, which includes Refs. [35,36,48,49].

[48] S. Aaronson and D. Gottesman, Phys. Rev. A 70, 052328 (2004).

[49] W. Dür, G. Vidal, and J. I. Cirac, Phys. Rev. Lett. 89, 057901 (2002).

[50] M. Van den Nest, J. Dehaene, and B. De Moor, Phys. Rev. A 69, 022316 (2004).

[51] D. P. Kingma and J. Ba, arXiv:1412.6980.

[52] J. Stokes, J. Izaac, N. Killoran, and G. Carleo, Quantum 4, 269 (2020).

[53] J. Stokes, J. Izaac, N. Killoran, and G. Carleo, SIAM J. Optim. 17, 188 (2006).

[54] C. E. Rasmussen, in Gaussian Processes in Machine Learning, edited by O. Bousquet, U. von Luxburg, and G. Rätsch, Lecture Notes in Computer Science Vol. 3176 (Springer, Berlin, Heidelberg, 2004).

[55] C. E. Rasmussen, in Summer School on Machine Learning (Springer, New York, 2003), pp. 63-71.

[56] P. I. Frazier, arXiv:1807.02811.

[57] M. Cerezo, A. Arrasmith, R. Babbush, S. C. Benjamin, S. Endo, K. Fujii, J. R. McClean, K. Mitarai, X. Yuan, L. Cincio, and P. J. Coles, arXiv:2012.09265.

[58] A simple way to get the same operation within the circuit framework is to use an ancilla qubit in place of the auxiliary qubit in the graph and act with $C X$ gates between the ancilla and the output qubits.

[59] A. Y. Kitaev, Ann. Phys. (Amsterdam) 303, 2 (2003).

[60] N. Klco, E. F. Dumitrescu, A. J. McCaskey, T. D. Morris, R. C. Pooser, M. Sanz, E. Solano, P. Lougovski, and M. J. Savage, Phys. Rev. A 98, 032331 (2018).

[61] C. J. Hamer, W. Zheng, and J. Oitmaa, Phys. Rev. D 56, 55 (1997).

[62] E. A. Martinez, C. A. Muschik, P. Schindler, D. Nigg, A. Erhard, M. Heyl, P. Hauke, M. Dalmonte, T. Monz, P. Zoller et al., Nature (London) 534, 516 (2016).

[63] C. Muschik, M. Heyl, E. Martinez, T. Monz, P. Schindler, B. Vogell, M. Dalmonte, P. Hauke, R. Blatt, and P. Zoller, New J. Phys. 19, 103020 (2017).

[64] F. Arute, K. Arya, R. Babbush, D. Bacon, J. C. Bardin, R. Barends, S. Boixo, M. Broughton, B. B. Buckley, D. A. Buell et al., Science 369, 1084 (2020).

[65] S. Coleman, Ann. Phys. (N.Y.) 101, 239 (1976).

[66] D. Yang, G. S. Giri, M. Johanning, C. Wunderlich, P. Zoller, and P. Hauke, Phys. Rev. A 94, 052321 (2016).

[67] W. Asavanant, Y. Shiozawa, S. Yokoyama, B. Charoensombutamon, H. Emura, R. N. Alexander, 
S. Takeda, J.-i. Yoshikawa, N. C. Menicucci, H. Yonezawa et al., Science 366, 373 (2019).

[68] M. V. Larsen, X. Guo, C. R. Breum, J. S. NeergaardNielsen, and U. L. Andersen, Science 366, 369 (2019).

[69] K. Tiurev, P. L. Mirambell, M. B. Lauritzen, M. H. Appel, A. Tiranov, P. Lodahl, and A. S. Sørensen, arXiv:2007.09298.

[70] K. Tiurev, M. H. Appel, P. L. Mirambell, M. B. Lauritzen, A. Tiranov, P. Lodahl, and A. S. Sørensen, arXiv:2007.09295.
[71] I. Schwartz, D. Cogan, E. R. Schmidgall, Y. Don, L. Gantz, O. Kenneth, N. H. Lindner, and D. Gershoni, Science 354, 434 (2016).

[72] T. Bastin, C. Thiel, J. von Zanthier, L. Lamata, E. Solano, and G. S. Agarwal, Phys. Rev. Lett. 102, 053601 (2009).

[73] L. Funcke, T. Hartung, K. Jansen, S. Kühn, and P. Stornati, arXiv:2011.03532. 